\title{
Evaluation of nocturnal heart rate variability for strenuous exercise day using wearable photoelectric pulse wave sensor
}

\author{
Yutaka Yoshida ${ }^{1, *}$, Emi Yuda $^{2}$, Kiyoko Yokoyama', Junichiro Hayano ${ }^{2}$ \\ ${ }^{1}$ Nagoya City University Graduate School of Design and Architecture, Nagoya, Japan \\ ${ }^{2}$ Department of Medical Education, Nagoya City University Graduate School of Medical Science, Nagoya, Japan
}

In this study, we examined whether or not the strenuous exercise in the evening change nocturnal heart rate variability (HRV) and recovers. Subjects were 8 healthy men belonging to the mature futsal team lage: $35 \pm 3$ years) and Futsal was held from 5:00 p.m. to 6:30 p.m. on Sunday. Pulse waves during sleeping were measured at home on exercise day and on control day. The mean pulse interval (MPI), standard deviation pulse interval (SDPI), low frequency component (LF, 0.04-0.15 Hz) , high frequency component $(\mathrm{HF}, 0.15-0.45 \mathrm{~Hz})$ and the ratio of $\mathrm{LF}$ to $\mathrm{HF}$ (LF/HF) were calculated from pulse interval time series every $30 \mathrm{~min}$. As a result, MPI, SDPI, LF, HF of exercise day were significantly lower than control day $(P<0.0001)$. LF/HF of exercise day was significantly higher than control day $(P<0.05)$. Regarding change of HRV for every $30 \mathrm{~min}$, MPI $(P<0.05)$, SDPI $(P=0.0003)$, LF $(P=0.0038)$, HF $(P<0.05)$ were observed significant changes. MPI and HF before wake-up did not reach the level of control day. It is thought that strenuous exercise in the evening promotes sympathetic nervous activity during night sleep and suggesting that pulse rate and HRV have not recovered by the wake-up time.

Keywords: Pulse waves, Nocturnal heart rate variability, Autonomic nervous system, Strenuous exercise, Recovery

\section{INTRODUCTION}

Physical activity has been reported to control cardiovascular risk factors such as obesity, diabetes mellitus, hypertension, and so on (Dhutia and Sharma, 2015). During the leisure time, the mortality rate of people with high physical activity is lower than the mortality rate of people with low physical activity (Arem et al., 2015; Liu et al., 2018). According to World Health Statistics 2016, Japan's healthy life expectancy is the world's No. 1 (83.7 years), and relationship between health life expectancy (World Health Organization, 2016) and heart rate variability (HRV) has also been reported from Japanese Holter electrocardiogram big data (Yuda et al., 2017). HRV reflects autonomic nervous activity, and decline of HRV is recognized as cardiovascular risk and mortality risk (Task Force of the European Society of Cardiology and the North American Society of Pacing and Electrophysiology,

\section{6).}

Exercise reduces HRV, but changes nocturnal HRV are affected by exercise habits, exercise intensity, time zones. In the previous study, walking and jogging for healthy young during $30 \mathrm{~min}$ in the morning did not have a significant influence nocturnal HRV (Yuda et al., 2018). Other studies have reported that moderate exercise and marathon in the afternoon (Myllymäki et al., 2012), and strong exercise in the evening from 6:00 p.m. increase nocturnal heart rate (HR) and lower nocturnal HRV (Hynynen et al., 2010). Generally, although exercise is a beneficial activity, overworking can stress the body and sometimes reduce nocturnal HRV (Driver and Taylor, 2000). Therefore, monitoring the recovery of nocturnal HRV leads to the determination of an appropriate exercise protocol.

In this study, we examined whether or not the strenuous exercise in the evening change nocturnal HRV and recovers. Noctur-
${ }^{*}$ Corresponding author: Yutaka Yoshida (iD https://orcid.org/0000-0001-5676-3048 Nagoya City University Graduate School of Design and Architecture, 2-1-10, Kita Chikusa, Chikusa-ku, Nagoya 464-0083, Japan

Tel: +81-52-721-5439, Fax: +81-52-721-3110, E-mail: yoshida@sda.nagoya-cu.ac.jp Received: June 24, 2018 / Accepted: July 24, 2018
This is an Open Access article distributed under the terms of the Creative Commons Attribution Non-Commercial License (http://creativecommons.org/licenses/by-nc/4.0/) which permits unrestricted non-commercial use, distribution, and reproduction in any medium, provided the original work is properly cited. 
nal HRV on holiday exercise day and weekday working day was analyzed using wearable photoelectric pulse wave sensor for amateur futsal team in their 30 s.

\section{MATERIALS AND METHODS}

\section{Subjects}

Subjects were 8 healthy men belonging to the mature futsal team (mean \pm standard deviation: age, $35 \pm 3$ years; height, $172 \pm$ $4 \mathrm{~cm}$; weight, $68 \pm 6 \mathrm{~kg}$; body mass index, $22.9 \pm 1.2 \mathrm{~kg} / \mathrm{m}^{2}$ ). The present study was performed according to the protocol that was approved by the Institutional Review Board of Nagoya City University Graduate School of Medical Sciences and Nagoya City University Hospital (approval number: 60160073).

\section{Experiment protocol}

Futsal was held from 5:00 p.m. to 6:30 p.m. on Sunday. The game format was a round robin of four teams, and the game time was $7 \mathrm{~min}$. Subjects took a rest for $7 \mathrm{~min}$ after the game and played the next game. Subjects repeated round robin two sets. However, for the convenience of the game combination, each team played two consecutive games at least once.

\section{Measurements}

Before the game started, the activity meter (Lifecorder GS, Suzuken Co., Ltd., Nagoya, Japan) was worn on the waist and the energy expenditure (EE) (kcal) of the physical activity during the game was measured. Using the wearable photoelectric pulse wave sensor, pulse waves during sleeping was measured at home on exercise day and on Wednesday (control day) of after 3 days too. Washout of exercise effect was 3 days. Also, the EE of control day was measured from wake-up time to bedtime, and refrained from drinking alcohol on both exercise day and control day.

\section{Wearable photoelectric pulse wave sensor}

Pulse wave and body movement acceleration during sleep were measured using the wearable photoelectric pulse wave sensor with built-in 3-axis acceleration sensor (APM, prototype, Suzuken Co. Ltd., Nagoya, Japan). External dimensions are W52×D46×H21 $\mathrm{mm}$, mass is $46 \mathrm{~g}$, pulse wave is detected with LED (peak emission wavelength $570 \mathrm{~nm}$ ) and photodetector. Piezoresistive type was used for 3-axis acceleration sensor (dynamic range, $\pm 3 \mathrm{G}$ ). Sampling frequency is $32 \mathrm{~Hz}$, pulse wave can be continuously recorded for $12 \mathrm{hr}$ by the lithium ion battery.

\section{Analysis of pulse wave and 3-axis acceleration}

After applying complex demodulation (CDM) to the pulse wave signal (Hayano et al., 1994), pulse interval time series was calculated at $2 \mathrm{~Hz}$ using pulse interval demodulation which directly extracts the instantaneous pulse interval as a continuous function from the pulse wave signal (Hayano et al., 2005). Fig. 1 shows pulse interval time series, pulse wave signal and 3 -axis ac-

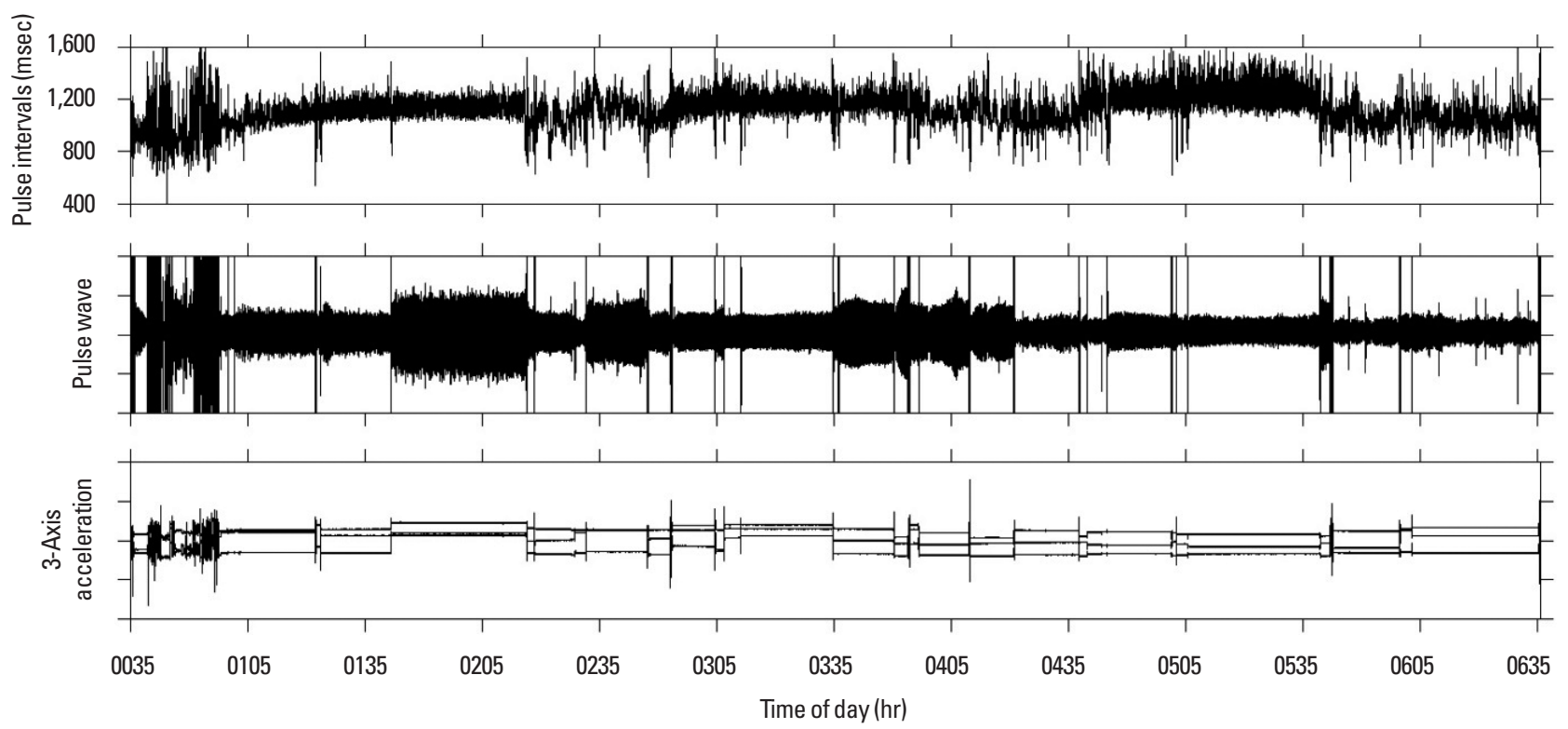

Fig. 1. Calculation of pulse interval time series using pulse interval demodulation and 3-axis acceleration. Measurement time is 0:35 a.m.-6:35 a.m., bed time is 1:00 a.m., wake-up time is 6:30 a.m. Upper panel: pulse interval time series. Middle panel: original pulse wave time series. Lower panel: 3-axis acceleration. 
celeration during night sleep. The mean pulse interval (MPI) and standard deviation pulse interval (SDPI) were calculated from the pulse interval time series. In addition, as frequency analysis, amplitude of the low frequency component (LF, 0.04-0.15 Hz) corresponding to $\mathrm{HRV}$, amplitude of the high frequency component (HF, 0.15-0.45 Hz) and the ratio of LF to HF (LF/HF) were calculated using CDM. All indices were calculated as calculation window for $30 \mathrm{~min}$. Bedtime was defined as the time when the change of 3-axis acceleration disappeared. Wake-up time was defined as the time which the acceleration change occurred when subjects woke up.

\section{Statistical analysis}

We used SAS 9.1 (SAS Institute Inc., Cary, NC, USA) for statistical analysis. To evaluate changes in HRV during night sleep at exercise day and control day, we used the mixed model of twoway analysis of variance with repeated measurements. Fixed effects were condition (exercise and control) and sleep phase (every 30 min), random effect was subject. $P<0.05$ was considered to be
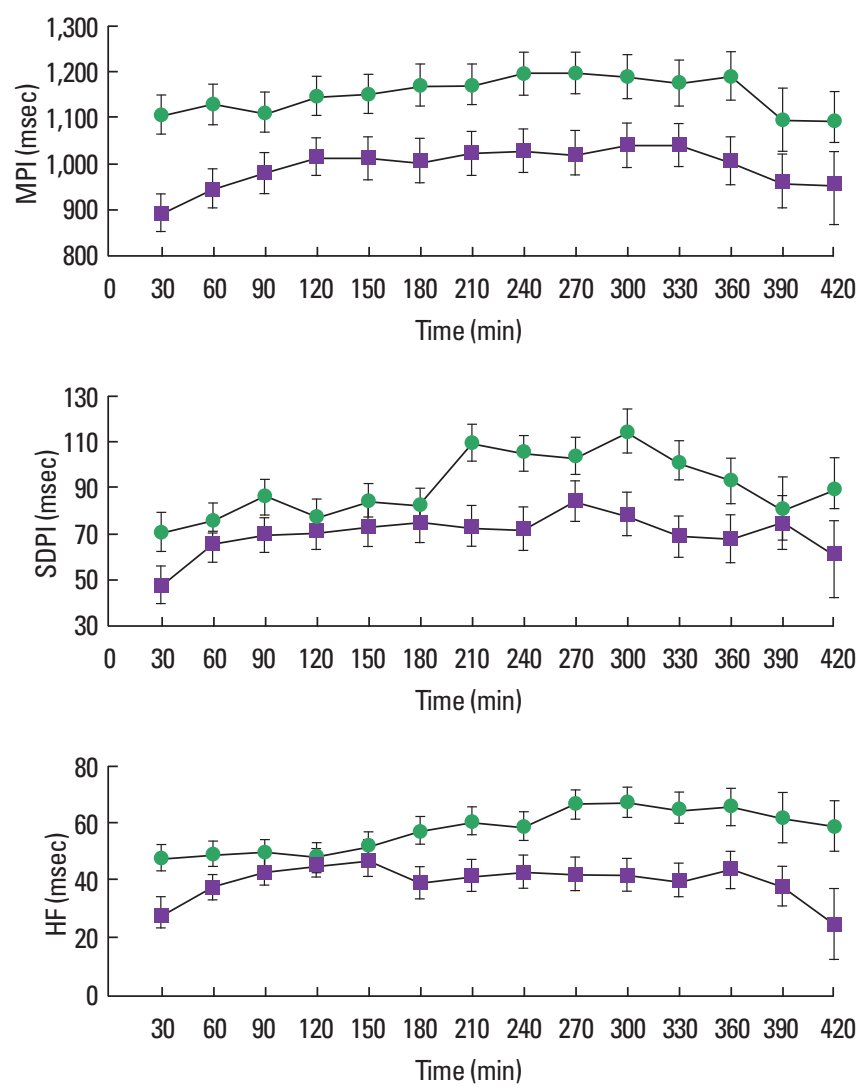

statistically significant.

\section{RESULTS}

Fig. 2 shows change of HRV indices during night sleep (mean \pm standard error). MPI, SDPI, LF, HF of exercise day were significantly lower than those of control day $(P<0.0001)$. LF/HF of exercise day was significantly higher than that of control day $(P<$ 0.05). Regarding change of HRV for sleep phase, MPI $(P<0.05)$, SDPI $(P=0.0003), \operatorname{LF}(P=0.0038), \mathrm{HF}(P<0.05)$ were observed significant changes. No interaction was observed between condition and sleep phase in all indices. EE during exercise was $247 \pm$ $88 \mathrm{kcal}$, EE on control day was $207 \pm 76 \mathrm{kcal}$.

\section{DISCUSSION}

In this study, to examine whether or not the strenuous exercise in the evening changes nocturnal HRV and recovers, we compared exercise day with control day. MPI, SDPI, LF, and HF of ex-
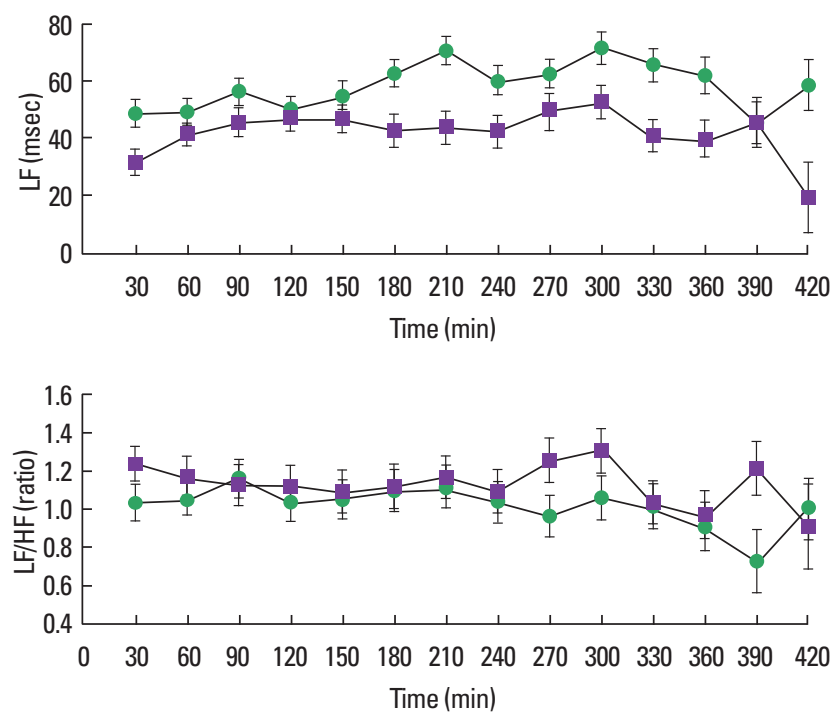

- Control day Exercise day

Fig. 2. Change of heart rate variability indices during sleep calculated using complex demodulation. Subjects were 8 healthy men. Sleeping time of exercise day was $362 \pm 43$ min, Sleeping time of control day was $347 \pm 70$ min (mean \pm standard deviation). MPI, mean pulse intervals; SDPI, pulse intervals of standard division; LF, low-frequency component amplitude; HF, high-frequency component amplitude; LF/HF, LF-to-HF component power ratio. 
ercise day were significantly lower than control day. These gentle increases were observed for $120 \mathrm{~min}$ after going to bed, after that, variability was suppressed, MPI and HF did not reach the level of control day. HF of HRV reflects cardiac parasympathetic activity, and $\mathrm{LF} / \mathrm{HF}$ reflects cardiac sympathetic nerve activity. Since HF decreases and LF/HF increases as compared to the control day, it is thought that strenuous exercise in the evening promotes sympathetic nervous activity during night sleep and suggesting that pulse rate and HRV have not recovered by the wake-up time.

As limitations of experiments, we did not measure maximum oxygen intake in this experiment, but instead we measured the amount of physical activity during exercise and control day using activity meter. The experimental protocol of this study carried out an intermittent exercise load of about 90 min repeated 6 times for 7-min futsal and 7-min break. The actual movement time was about $42 \mathrm{~min}$, and the amount of physical activity at this time was $247 \pm 88 \mathrm{kcal}$. The result of recovery delay with increase of HR and decrease of HRV during night sleep for strenuous exercise in the evening are consistent with the study results of Myllymaki et al. (2012).

According to a report of Myllymaki et al. (2012), as a result of exercise from 6:00 p.m. in experimental protocols of exercise intensity (maximum oxygen intake, 45\%, 60\%, 75\%) and exercise time (30 min, $60 \mathrm{~min}, 90 \mathrm{~min}$ ) for healthy men in their 30s, nocturnal HR increased with exercise intensity than control day and nocturnal HRV was lower after the 90 min exercise day compared to control day. Hynynen et al. (2010) reported that nocturnal HR was increased and nocturnal HRV was lower than control day in an experiment that combined a moderate exercise of $50 \mathrm{~min}$ in the afternoon and a marathon of $3.5 \mathrm{hr}$.

Although these previous studies are results using electrocardiogram measurement, our study is the result of using a wrist watch type wearable photoelectric pulse wave sensor which can be measured conveniently in daily life. This suggests the possibility of self-monitoring autonomic nervous activity by using wearable photoelectric pulse wave sensor in daily life and it is an important research material for popularization of wearable photoelectric pulse wave sensor. Strenuous exercise in the evening for healthy men in their 30s, nocturnal pulse rate, reduces nocturnal HRV, and did not recover by the wake-up time.

\section{CONFLICT OF INTEREST}

No potential conflict of interest relevant to this article was reported.

\section{ACKNOWLEDGMENTS}

This study was performed as a part of the collaborative studies with Shinsakae Futsal Arena, Nagoya, Japan.

\section{REFERENCES}

Arem H, Moore SC, Patel A, Hartge P, Berrington de Gonzalez A, Visvanathan K, Campbell PT, Freedman M, Weiderpass E, Adami HO, Linet MS, Lee IM, Matthews CE. Leisure time physical activity and mortality: a detailed pooled analysis of the dose-response relationship. JAMA Intern Med 2015;175:959-967.

Dhutia H, Sharma S. Playing it safe: exercise and cardiovascular health. Practitioner 2015;259:15-20, 22.

Driver HS, Taylor SR. Exercise and sleep. Sleep Med Rev 2000;4:387-402.

Hayano J, Barros AK, Kamiya A, Ohte N, Yasuma F. Assessment of pulse rate variability by the method of pulse frequency demodulation. Biomed Eng Online 2005;4:62.

Hayano J, Taylor JA, Mukai S, Okada A, Watanabe Y, Takata K, Fujinami T. Assessment of frequency shifts in R-R interval variability and respiration with complex demodulation. J Appl Physiol (1985) 1994;77:28792888.

Hynynen E, Vesterinen V, Rusko H, Nummela A. Effects of moderate and heavy endurance exercise on nocturnal HRV. Int J Sports Med 2010;31: 428-432.

Liu Y, Shu XO, Wen W, Saito E, Rahman MS, Tsugane S, Tamakoshi A, Xiang YB, Yuan JM, Gao YT, Tsuji I, Kanemura S, Nagata C, Shin MH, Pan WH, Koh WP, Sawada N, Cai H, Li HL, Tomata Y, Sugawara Y, Wada K, Ahn YO, Yoo KY, Ashan H, Chia KS, Boffetta P, Inoue M, Kang D, Potter JD, Zheng W. Association of leisure-time physical activity with total and cause-specific mortality: a pooled analysis of nearly a half million adults in the Asia Cohort Consortium. Int J Epidemiol 2018 Feb 27 [Epub]. https://doi.org/10.1093/ije/dyy024.

Myllymäki T, Rusko H, Syväoja H, Juuti T, Kinnunen ML, Kyröläinen H. Effects of exercise intensity and duration on nocturnal heart rate variability and sleep quality. Eur J Appl Physiol 2012;112:801-809.

Task Force of the European Society of Cardiology and the North American Society of Pacing and Electrophysiology. Heart rate variability: standards of measurement, physiological interpretation and clinical use. Circulation 1996;93:1043-1065.

World Health Organization. World Health Statistics 2016: Monitoring health for the SDGs. Geneva (Switzerland): World Health Organization; 2016.

Yuda E, Furukawa Y, Yoshida Y, Hayano J, ALLSTAR Research Group. Association between regional difference in heart rate variability and 
inter-prefecture ranking of healthy life expectancy: ALLSTAR big data project in Japan. In: Jung JJ, Kim P, editors. Big data technologies and applications. Lecture notes of the institute for computer sciences, social informatics and telecommunications engineering, 2017;194:23-28.
Yuda E, Moriyama Y, Mori T, Yoshida Y, Kawahara M, Hayano J. Acute effects of endurance exercise on nocturnal autonomic functions in sedentary subjects: a pilot study. J Exerc Rehabil 2018;14:113-117. 